\title{
Brief Ataxia Rating Scale
}

National Cancer Institute

\section{Source}

National Cancer Institute. Brief Ataxia Rating Scale. NCI Thesaurus. Code C98367.

A validated instrument to assess ataxia using five criteria for evaluation: Gait, Knee-tibia test, Finger-to-nose test, Dysarthria, and Occulomotor abnormalities. 